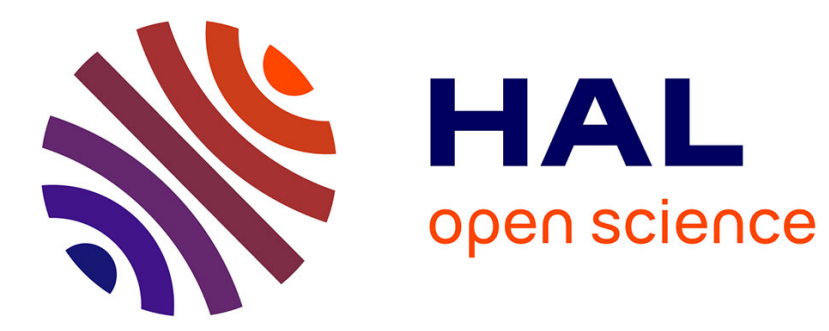

\title{
WHAT ODD EVEN EFFECTS MEAN IN LOW ENERGY FISSION?
}

\author{
M. Montoya
}

\section{To cite this version:}

M. Montoya. WHAT ODD EVEN EFFECTS MEAN IN LOW ENERGY FISSION?. International Workshop on Semiclassical and Phase Space Approaches to the Dynamics of the Nucleus, 1987, Aussois, France. pp.C2-353-C2-357, 10.1051/jphyscol:1987254 • jpa-00226523

\section{HAL Id: jpa-00226523 https://hal.science/jpa-00226523}

Submitted on 1 Jan 1987

HAL is a multi-disciplinary open access archive for the deposit and dissemination of scientific research documents, whether they are published or not. The documents may come from teaching and research institutions in France or abroad, or from public or private research centers.
L'archive ouverte pluridisciplinaire HAL, est destinée au dépôt et à la diffusion de documents scientifiques de niveau recherche, publiés ou non, émanant des établissements d'enseignement et de recherche français ou étrangers, des laboratoires publics ou privés. 
WHAT ODD EVEN EFFECTS MEAN IN LOW ENERGY FISSION ?

\author{
M. MONTOYA $(1)$ \\ Institut de Physique Nucléaire, BP I, F-91406 orsay Cedex, \\ France
}

Résumé. Les effets pair-impair dans les quantités de fragments dans ${ }^{233} U\left(n_{t h}, f\right),{ }^{235} U$ $\left(n_{t h}, f\right)$ and ${ }^{239} P u\left(n_{t h}, f\right)$ sont interprétés dans le cadre d'un mécanisme de brisure statistique de paires de nucléons dans la descente du point selle au point de scission. La probabilité pour qu'aucune paire ne soit brisée est de l'ordre de 1 pour 1000 . Pour ${ }^{235} \mathrm{U}$ et ${ }^{239} \mathrm{Pu}$, on estime à 3.5 et \& les nombres moyens de paires brisées. Les variances des distributions respectives sont de 1.4 et 1.3. La probabilité pour qu'une paire brisée soit de protons (neutrons) est proportionnelle au nombre de paires de protons (neutrons) dans le système fissile.

\begin{abstract}
Odd even effects in fragment quantities from ${ }^{233} U\left(n_{t h}, f\right),{ }^{236} U\left(n_{t h}, f\right)$ and ${ }^{239} \mathrm{Pu}\left(n_{t h}, f\right)$ are explained in terms of a statistical pair breaking mechanism during the descent from saddle to scission point. The probability that no pair will be broken is of the order of 1 per 1000. For ${ }^{233} U\left(n_{t h}, f\right)$ and ${ }^{235} U\left(n_{t h}, f\right)$, the average number of broken pair are 3.5 and 4., respectively, and the variances of the corresponding distributions are 1.4 and 1.3. The probability for one broken pair to be of protons (neutrons) is proportional to the numbers of proton (neutron) pairs in the system.
\end{abstract}

\title{
1. Introduction
}

Odd even effects in fragment charge distribution $[1,2,3,4,5], \delta Z\left({ }^{Z} Y_{e}-{ }^{Z} Y_{o}\right.$, where ${ }^{z} Y_{e}$ and ${ }^{z} Y_{o}$ are the yields of fission fragments having even and odd charge, respectively), neutron number distribution $[2,4], \delta N$ (defined similarly to $\delta Z$ ), in low energy fission of even-even nuclei have been measured by several authors. Proton odd even effect in average fragment kinetic energy, $\delta \bar{E}\left({ }^{Z} E_{e}-{ }^{Z} E_{o}\right.$, where ${ }^{Z} E_{e}$ and ${ }^{Z} E_{o}$ are the kinetic energies of even-Z and odd-Z fragments, respectively), has also been observed $[3,4,5]$.

Those odd-even effects were explained in terms of proton pair breaking in the system during the descent from saddle to scission and supposed to be a proof that a fraction of fission events corresponds to a superfluid mechanism, i.e. occurs without any pair breaking. In the first interpretations of odd even effects proton and neutron pairs were treated independently $[2,3,4,5]$.

The increasing of $\delta Z$ and $\delta N$ as a function of fragment kinetic energy [4] suggested that a strong hindrance of odd mass (even Z-odd $\mathrm{N}$ or odd Z-even $\mathrm{N}$ ) would be found at higher fragment kinetic energies. However experimental results show that even at high fragment kinetic energies odd mass numbers are competitive with even mass numbers [6]. In order to understand this result it was suggested to take into account correlations between those effects [7].

A combinatorial model in which proton and neutron pair breaking events are coherent was developed by $\mathrm{H}$. Nifenecker et al. [8]. In order to reproduce experimental odd even effects, these authors assume that a large fraction of broken nucleon pairs had to occur at scission and a small fraction during the descent from saddle to scission point. Nevertheless the hypothesis of early pair breaking (before scission) is also compatible with experimental results on odd even effects [9,10].

\footnotetext{
(1) On leave from the Facultad de Ciencias, Universidad Nacionale de Ingeniería, Apt. 1301, Lima, Peru
} 


\section{More than one broken pair}

One can show that a large fraction of fragmentations has at least two broken nucleon pairs. For that one firstly remembers that from definitions of $\delta Z, \delta N$ and $\delta A$ one had deduced that the probability of odd- $Z$ and odd- $N$ fragmentations is given by the relation [9]

$$
P_{\circ o}=\frac{1}{4}(1+\delta A-\delta Z-\delta N)
$$

If one makes the hypothesis of statistical pair breaking ( $S P B$ ), i.e. the nucleons from a broken pair will end up independently in the complementary fragments (Notice that if pair breaking occurs before scission the hypothesis $S P B$ is true) then $\delta Z, \delta N$ and $\delta A$ are the fraction of events for which no proton, neutron and nucleon pair, respectively, has been broken. Moreover the fraction of events in which one proton pair and one neutron pair were be broken is

$$
P_{2}=4 \times P_{o o} \text {. }
$$

For ${ }^{235} U\left(n_{t h}, f\right)\left(\delta Z=0.237\right.$ and $\delta N=0.054$ [4]) one obtains $P_{o o}=0.70$, i.e. for $70 \%$ of fission events at least two nucleon pairs are broken.

\section{Average odd even effects}

One way to express a correlation between proton and neutron pair breaking is to assume a combinatorial pair breaking mechanism [8]. In that mechanism the probability for a nucleon pair to be broken, $q$, and the probability that a broken pair will be a proton one, $\epsilon$, are independent of the state of the system; and the total number of nucleon pairs available to be broken, $M$, depends on the fragment excitation energy. From the hypothesis of $S P B[9]$ one can show that [10]

$$
\begin{aligned}
\delta Z & =(1-q \epsilon)^{M},(a) ; \delta N=(1-q(1-\epsilon))^{M},(b) ; \delta A=(1-q)^{M},(c) ; \\
\delta^{Z} \bar{E} & =\frac{2 \delta Z}{1-\delta Z^{2}} M \Delta E \frac{1-\delta Z^{\frac{1}{M}}}{\delta Z^{\frac{1}{M}}}\left(\delta Z^{\frac{1}{M}}+\delta N^{\frac{1}{M}}-1\right) ;
\end{aligned}
$$

where $\delta E$ is the shift in fragment kinetic energy produced by each nucleon pair breaking. If one takes $\Delta E=2.5 \mathrm{MeV}$, from equations (3-4), using experimental odd even effects, one can estimate

Table 1:

\begin{tabular}{|l|r|r|r|r|r|r|}
\hline system & $\delta \bar{Z}$ & $\delta \bar{N}$ & $\delta^{2} \bar{E}$ & $\epsilon$ & $\bar{N}$ & $\sigma_{N}^{2}$ \\
\hline${ }^{235} U[4]$ & .237 & .054 & 0.7 & .34 & 3.5 & 1.4 \\
${ }^{239} P U[11]$ & .116 & .005 & 0.5 & .45 & 4.1 & 1.3 \\
\hline
\end{tabular}

the average number, $\bar{M}$, and the variance, $\sigma_{M}^{2}$, of the number of broken nucleon pairs distribution which are equivalent to $q\left(=1-\frac{\sigma_{M}^{2}}{M}\right)$ and $M\left(=\frac{\bar{M}}{q}\right)$ values. In table 1 , the results for ${ }^{235} U\left(n_{t h}, f\right)$ and ${ }^{239} P u\left(n_{t h}, f\right)$ are presented.

\section{Cold fission}

From equations ( $3-4)$ and parameters represented in Table 1 one obtains $\delta A=0.003$ and 0.001 from ${ }^{235} U\left(n_{t h}, f\right)$ and ${ }^{239} P u\left(n_{t h}, f\right)$, respectively. This odd-even effect can only be measured in the so-called cold fission, i.e. fragmentation at high fragment kinetic energy or low excitation energy. One way to do this is to select fragments whose $T K E$-values are higher than a defined threshold, $K$. Odd even effect in the yield of the masses $A, A+1, A+2$, (A being even) is defined by

$$
\delta A_{(A, K)}=\frac{\frac{Y_{A}+Y_{A+2}}{2}-Y_{A+1}}{\frac{Y_{A}+Y_{A+2}}{2}+Y_{A+1}} .
$$


In Tables 2 and 3 the experimental values of $\delta A_{(A, K)}$ from ${ }^{233} U\left(n_{t h}, f\right)$ and ${ }^{235} U\left(n_{t h}, f\right)$ are presented.(The data source is the same as the one used to obtain equiprobability lines in the $T K E$ and $A$ distribution in Ref. [12]). Taking into account that $\delta A=0$ at lower $T K E$, one can estimate that the average $\delta A$ is of the order of 0.001 .

Table 2: ${ }^{233} U\left(n_{t h}, f\right)$

\begin{tabular}{|l|r|r|r|r|r|}
\hline$A$ & $T K E \geq$ & $Y_{A}$ & $Y_{A+1}$ & $Y_{A+2}$ & $\delta A_{(A)}$ \\
\hline 102 & $204 \mathrm{MeV}$ & 40 & 20 & 20 & 0.2 \\
95 & $193.8 \mathrm{MeV}$ & 7 & 2 & 16 & 0.7 \\
92 & $190 \mathrm{MeV}$ & 10 & 3 & 15 & 0.6 \\
90 & $198.5 \mathrm{MeV}$ & 12 & 14 & 40 & 0.3 \\
86 & $179 . \mathrm{MeV}$ & 16 & 4 & 18 & 0.6 \\
\hline
\end{tabular}

Table 3: ${ }^{235} U\left(n_{t h}, f\right)$

\begin{tabular}{|l|r|r|r|r|r|}
\hline$A$ & $T K E \geq$ & $Y_{A}$ & $Y_{A+1}$ & $Y_{A+2}$ & $\delta A_{(A)}$ \\
\hline 100 & $203.8 \mathrm{MeV}$ & 14 & 8 & 10 & 0.2 \\
102 & $202.9 \mathrm{MeV}$ & 18 & 10 & 14 & 0.23 \\
104 & $198.8 \mathrm{MeV}$ & 18 & 10 & 30 & 0.4 \\
94 & $193.3 \mathrm{MeV}$ & 23 & 13 & 35 & 0.38 \\
84 & $176 . \mathrm{MeV}$ & 19 & 10 & 30 & 0.42 \\
\hline
\end{tabular}

Cold fragmentation was measured from ${ }^{233} U\left(n_{t h}, f\right),{ }^{235} U\left(n_{t h}, f\right)$ and ${ }^{239} P u\left(n_{t h}, f\right)$, by several authors (See $[6,13]$ and references therein). In order to interpret cold fission, fragment deformation and Coulomb energies, $D$ and $C$, were calculated for several scission configurations. The comparison of the potential energy, $P(=C+D)$, with the $Q$-value suggests that cold fragmentation corresponds to very compact scission configurations in which pre-scission fragment kinetic energy, $T K E_{0}$, and intrinsic excitation energy, $X$, are equal to zero. This is compatible with the hypothesis that cold fission occurs near the saddle point.

J. Berger et al. have calculated a potential energy surface to describe low energy fission processes [14]. This surface presents a fission valley and a fusion valley separated by a ridge. Cold fission would occur by tunneling from the fission to the fusion valley at the beginning of the descent into the fission valley. Those very few fragmentations $(0.1 \%)$ in which no pair breaking had occured would reach higher $T K E$-values than the others as it was clearly observed by $\mathrm{J}$. Trochon et al. [15].

\section{Odd even effects as a function of kinetic energy}

Let's assume fission as a sequence of two steps. Step 1: the system goes down into the fission valley heating, and deforming. Step 2: the system goes from the fission valley to the fusion valley at the scission point. The lower the exit point, $S$, will be in the fission valley the higher the excitation energy, $X$, will be the higher will be the number of available pairs for breaking, $M$, and the lower will be the final fragment kinetic energy $(T K E=Q-X)$.

Let $\delta_{\left(E_{L}\right)}$ (which is the fraction of events for which no pair has been broken) the (proton, neutron or nucleon) odd even effect of fragments having a kinetic energy $E_{L}$. The odd even effects for later fragmentations, corresponding to the kinetic energy $E_{L}-\Delta E_{L}$, will be given by the relations

$$
\frac{\Delta \delta Z}{\delta Z \Delta E_{L}}=\alpha_{p},(a) ; \frac{\Delta \delta N}{\delta N \Delta E_{L}}=\alpha_{n},(b) ; \frac{\Delta \delta A}{\delta A \Delta E_{L}}=\alpha_{p n},(c)
$$

where $\alpha_{p n}=\alpha_{p}+\alpha_{n}$ and where $\alpha_{p}, \alpha_{n}$ and $\alpha_{p n}$ (which are the probabilities that a new proton, neutron and nucleon pair, respectively, will be broken by diminishing the selected $E_{L}$ of $\Delta E_{L}$ ), are assumed to be constant.

The function obeying those relations is

$$
\delta=\delta_{\text {ave }} e^{\alpha\left(E_{L}-E_{0}\right)} .
$$

In Table 4 one presents parameters of the function fitting experimental results from ${ }^{235} U\left(n_{t h}, f\right)$ and ${ }^{239} P u\left(n_{t h}, f\right)$. Notice that $\frac{\alpha_{p}}{\alpha_{n}}=\frac{Z}{N}$, where $Z$ and $N$ are the number of protons and neutrons in the fissionable nuclei, i.e. the probability that a broken pair will be a proton one is proportional to the number of proton pairs in the system. In Fig.1 the curves fitting the experimental values on odd-even effects obtained by H.-G. Clerc et al. [13]. 
Table 4:

\begin{tabular}{|l|r|r|r|r|r|r|}
\hline system & $\alpha_{p}$ & $\alpha_{n}$ & $E_{0 p}$ & $E_{0_{n}}$ & $\frac{\alpha_{p}}{\alpha_{n}}$ & $\frac{Z}{N}$ \\
\hline${ }^{235} U[4]$ & .0303 & .0475 & 100. & 100 & .638 & .639 \\
$230 P u[11]$ & .0419 & .0641 & 101. & 101. & .654 & .644 \\
\hline
\end{tabular}

From definitions one can deduce the relations

$$
\delta^{Z} \bar{E}=\frac{2 \delta Z_{a v e}}{1-\delta Z_{a v e}^{2}} \alpha_{p} \sigma_{E_{L}}^{2},(a) ; \delta^{N} \bar{E}=\frac{2 \delta N_{a v e}}{1-\delta N_{a v e}^{2}} \alpha_{n} \sigma_{E_{\Sigma}}^{2},(b) ; \delta^{A} \bar{E}=\frac{2 \delta A_{a v e}}{1-\delta A_{a v e}^{2}} \alpha_{p n} \sigma_{E_{\Sigma}}^{2},(c)
$$

where $\sigma_{E_{L}}^{2}$ is the variance of the $E_{L}$ distribution. Notice that if $\delta Z$, is constant as a function of $E_{L}, \delta^{Z} \bar{E}_{L}=0$. Using experimental results of $\sigma_{E_{L}}^{2}\left(25 . \mathrm{MeV}^{2}\right)$ and $\delta Z_{\text {ave }}(0.237)[4]$ and $\alpha_{p}=0.0303$ one obtains $\delta \bar{E}_{L}=0.4$ for ${ }^{235} U\left(n_{t h}, f\right)$ which agrees with experimental result.
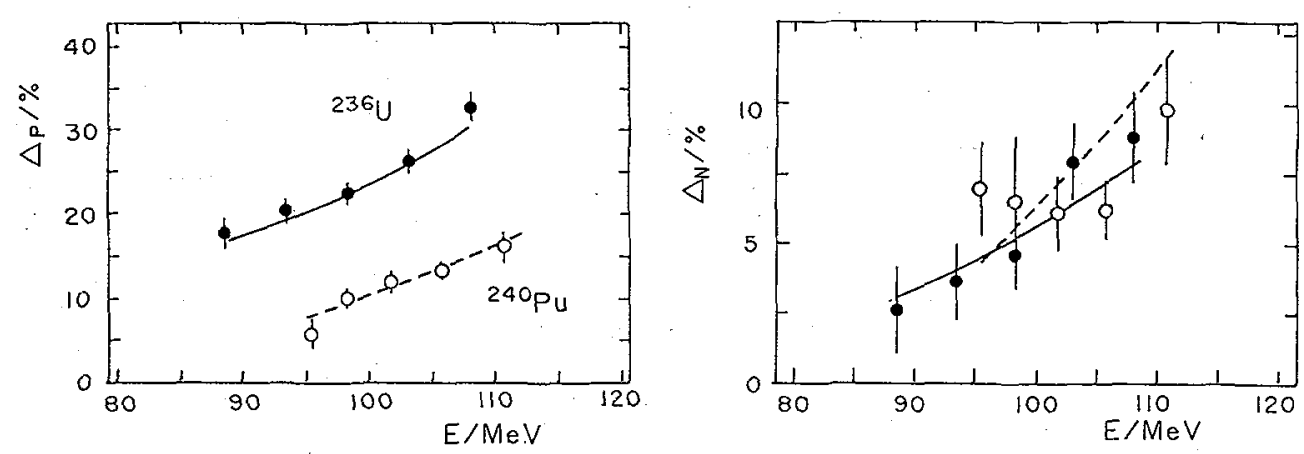

Fig. 1 Experimental (From H.-G. Clerc et al. data (13]) and calculated (see text.) values of proton (left) and neutron (right) odd-even effects as a function of light fragment kinetic energy from ${ }^{235} U\left(n_{t h}, f\right)$ and ${ }^{239} P u\left(n_{t h}, f\right)$.

\section{Conclusion}

The total fragment mass, charge and kinetic energy distribution from low energy fission of even-even heavy nuclei is compatible with the hypothesis of statistical nucleon pair breaking, i.e. the nucleons from a broken pair will end independently in the complementary fragments. If pair breaking occurs during the descent from saddle to scission that hypothesis is true.

Cold fission presents negligible odd-even effect in mass distribution whose origin is fragmentations near the saddle point, where there is not enough excitation energy to break a pair.

The major part of fission events corresponds to scission points far from saddle point and the probability that no nucleon pair will be broken is approximately 1 per 1000 . The distributions of the number of broken pairs in ${ }^{235} U\left(n_{t h}, f\right)$ and ${ }^{239} P u\left(n_{t h}, f\right)$ correspond to average numbers of 3.5 and 4 . and variances of 1.4 and 1.3 .

The probability for one pair broken to be of protons (neutrons) is proportional to the number of proton (neutron) pairs present in the fissionable system.

\section{References}

[1] A.L. Wahl et al., Proc. IAEA Symp. Phys. Chem. Fission, 2nd, p. 19,Vienna,1969

2] H.-G. Clerc et al., Proc. IAEA Symp. Phys. Chem. Fission, 4th, p. ,Vienna, 1980

3) H.A. Nifenecker et al., Proc. IAEA Symp. Phys. and Chem. Fission, 4th, p. 35, Vienna,1980 
[4] W. Lang et al., Nucl. Phys. A 345 (1980) 34

5. G. Mariolopoulos et al., Nucl. Phys. A361 (1981) 213

6] C. Signarbieux et al., J. Physique Lett. 42 (1981) L437

7 M. Montoya, thesis, Université d'Orsay, Orsay, 1981

8] H. Nifenecker et al., Z.f. Phys. A308 (1982) 39

9] $M$. Montoya, J. Phys (Paris) 44 (1983) 785

10] M. Montoya J. Phys. (Paris) C6 (1984) 407

[11] C. Schmitt et al., Nul. Phys. A430 (1984) 21

[12] M. Montoya, Z. Phys. A-Atoms and Nuclei 319 (1984) 219

13] H.-G. Clere et al., Nucl. Phys. A452 (1986) 277

14] J.F. Berger et al., Nucl. Phys. A428 (1984) 389 c

15] J. Trochon et al., Int. Conf. Nuclear Data for Basic and Applied Science, Santa Fe, 1985. 